\title{
On a thermodynamic theory of rods with two temperature fields
}

\begin{abstract}
This paper presents a thermodynamic theory for elastic rods using the model of directed curves. In this model, the thin rod-like bodies are described as deformable curves with a triad of rigidly rotating vectors attached to each point. To account for the thermal effects in rods, we introduce two independent temperature fields: the absolute temperature field and the temperature deviation field. We present a complete derivation of the non-linear equations of thermoelastic rods, starting from the principles of thermodynamics. Finally, we prove the uniqueness of solution to the linearized equations of thermoelastodynamics for rods.
\end{abstract}

\section{Introduction}

Mechanics of rods, bars, beams, and other thin bodies for which two dimensions are much smaller in comparison to the third one has a long history of development, see [1-3]. Nowadays, the rod and beam structures are widely used in engineering. Mechanics of rods and beams is summarized in many books, see for example [4-11]. In the theory of rods, there are various approaches to derive the governing equations. The first was the so-called direct approach used by Euler (1744) in well-known elastica problem. Within this approach, the rod is modeled as an elastic curve, and the constitutive equations are written as for an one-dimensional (1D) continuum. This model was generalized by the Cosserat brothers [12], considering translations and rotations as independent variables, which is known now as the Cosserat curve. Since Euler's time the direct approach was applied to the derivation of basic equations of mechanics of rods in many works, see for example, the seminal papers by Ericksen and Truesdell [13], Green and Naghdi [14,15], DeSilva and Whitman [16], Kafadar [17],

Dedicated to Professor Hans Irschik on the occasion of his 60th birthday.

H. Altenbach $(\bowtie)$. V. A. Eremeyev

Fakultät für Maschinenbau, Institut für Mechanik, Otto-von-Guericke-Universität Magdeburg,

Universitätsplatz 2, 39106 Magdeburg, Germany

E-mail: holm.altenbach@ovgu.de

Tel.: +49-391-6718814

Fax: +49-391-6712863

M. Bîrsan

Department of Mathematics, University "A.I. Cuza" of Iaşi, 700506 Iaşi, Romania

E-mail: bmircea@uaic.ro

M. Bîrsan

Faculty of Civil Engineering and Architecture, Lublin University of Technology, 20-618 Lublin, Poland

V. A. Eremeyev

South Scientific Center of RASci \& South Federal University, Rostov on Don, Russia 344090

E-mail: eremeyev.victor@gmail.com 
Green et al. [18], and the books [4,7]. Another way to derive one-dimensional equations of rods is to use the thinness hypothesis and to apply a reduction procedure to three-dimensional (3D) thin rod-like bodies. This derivation can be performed by the application of kinematical or stress hypotheses, the integration over the rod cross-section, or within mathematical techniques like series expansions and asymptotic analysis, see for example, $[5,6,8,10,11,19]$. In relation to mechanics of rods and beam, it is worth mentioning the contribution of Hans Irschik in this field, see [20-27] where the elasto-plastic behavior, vibrations, non-homogeneity, thermal, and electric fields coupling are studied, in particular.

In most papers on rod mechanics, the purely mechanical theory is considered without derivation of rod thermodynamics. This paper presents a thermodynamic theory for rods with two temperature fields using the direct approach. Within the direct approach, we suggest the rod as a deformable Cosserat curve, the kinematics of which is described by independent translations and rotations fields. In addition, we introduce two temperature fields, that is, the rod-like temperature and its deviation. In this case, it is necessary to introduce two dual entropy fields.

Let us note that the thermomechanics of 3D continua is always based on the First and Second Law of Thermodynamics formulated as the energy balance equation and the Clausius-Duhem inequality [28]. Discussion on other extended thermodynamic approaches is given in [29-33]. However, in the construction of 1D thermodynamic relations for rods, one cannot simply transfer the notions of temperature, entropy, and energy from the $3 \mathrm{D}$ case to the $1 \mathrm{D}$ continuum $\mathcal{C}_{0}$. For rods, it is quite natural to introduce the temperature fields describing the 3D distribution of the temperature on the rod cross-section. The situation with the rod thermodynamics is quite similar to the thermodynamics of shells where various sets of surface fields responsible for the temperature are used and several formulations of the first and second law are discussed, see [35-37]. The thermomechanics of rods has been considered earlier by DeSilva and Whitman [16], Kafadar [17], Green and Naghdi [14,15] applying the axiomatic approach, and by Simmonds [38] on the basis of exact integration of 3D equations of non-linear thermoelasticity. From the thermodynamic point of view, the $1 \mathrm{D}$ and $2 \mathrm{D}$ structures such as rods and shells can be considered as examples of generalized thermomechanical models or models with internal variables discussed for the 3D case in [29-33].

To describe the thermomechanics of general rods, we shall employ the kinematical model of directed curves presented in $[4,5,9,39]$. In this approach, the thin rod-like bodies are modeled as deformable curves endowed with a triad of vectors (also called directors) attached to each point of the curve. This triad of directors rotate rigidly during deformation and give information about rotations of the rod's cross-sections. In other words, we consider the rod as a 1D Cosserat or micropolar continuum. In the case of isothermal deformation, the complete purely mechanical theory is given in $[5,9,39]$. In this paper, we extend the model of directed curves and present a thermodynamical theory capable of describing thermal effects in rods. In this purpose, we consider two independent temperature fields associated to each point of the deformable curve: the absolute temperature field and the temperature deviation field. The former is a mean value of the absolute temperature in the cross-section of the rod, while the latter measures the spatial variation of the temperature in the cross-section. We also introduce two dual entropy fields, that is, the mean entropy and the entropy deviation, and two related entropy fluxes.

We mention that the approach of directed curves can also be extended to account for:

- porosity effects in the mechanics of rods $[40,41]$, using the theory of thermoelastic materials with voids [42];

- functionally graded materials in the mechanics of rods [43] or

- electrical and magnetic fields, phase transitions, diffusion, and other coupled effects known in 3D continuum mechanics or in $2 \mathrm{D}$ shell theories.

The paper begins with a short expository section, which presents the kinematical model of directed curves. In Sect. 3, we formulate the basic laws of thermodynamics for rods with two temperature fields, and we deduce the balance equations in local forms. In Sect. 4, we introduce the constitutive assumptions for thermoelastic rods and obtain the reduced constitutive equations. The energy balance equation coincides with the formulation in [38] while the second thermodynamics law differs from [38] by additional terms describing the entropy fluxes. We derive two thermoconductivity equations for determination of rod-like temperature and its deviation. The structure of the constitutive tensors for general rods is also discussed. In Sect. 5, we derive the linearized equations of thermoelastic rods with two temperature fields and we prove the uniqueness of solution to the boundary-initial-value problems. The uniqueness property in the linear setting shows that the theory is well formulated. 


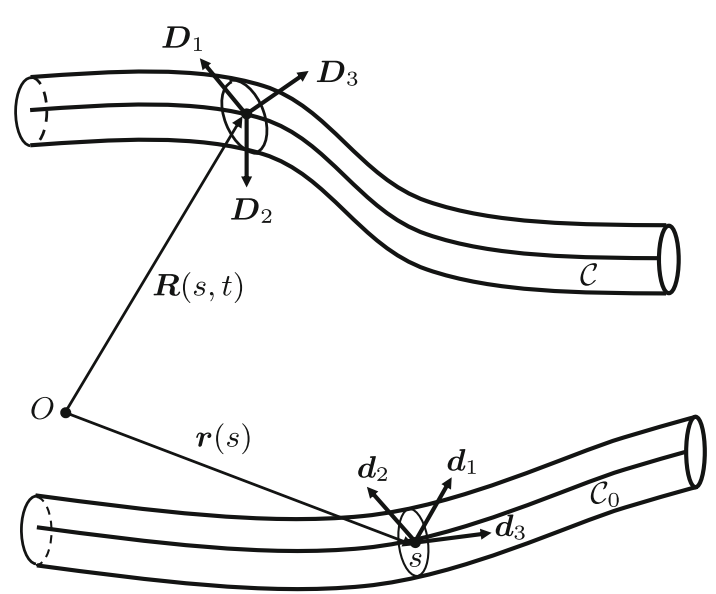

Fig. 1 Reference configuration $\mathcal{C}_{0}$ and deformed configuration $\mathcal{C}$ of the rod

\section{Kinematical model of directed curves}

Let us briefly present the kinematical model of directed curves. We denote by $\mathcal{C}_{0}$ the curve in the reference (initial undeformed) configuration and by $s$ the material coordinate along $\mathcal{C}_{0}$. The coordinate $s$ is taken to be the arclength parameter along $\mathcal{C}_{0}$. The reference configuration of the rod is defined by the vector fields (see Fig. 1)

$$
\boldsymbol{r}=\boldsymbol{r}(s), \quad \boldsymbol{d}_{i}=\boldsymbol{d}_{i}(s), \quad i=1,2,3, \quad s \in[0, l] .
$$

Here, $\boldsymbol{r}$ is the position vector of points on $\mathcal{C}_{0}$, the triad $\left\{\boldsymbol{d}_{1}, \boldsymbol{d}_{2}, \boldsymbol{d}_{3}\right\}$ specifies the 3 unit vectors (directors) orthogonal to each other, and $l$ is the length of the rod. The vector $\boldsymbol{d}_{3}$ is chosen tangent to the curve (i.e., $\boldsymbol{d}_{3}=\frac{\mathrm{d} \boldsymbol{r}}{\mathrm{d} s}$ ), while $\boldsymbol{d}_{1}$ and $\boldsymbol{d}_{2}$ are usually taken along the principal axes of inertia of the cross-section, and $\mathcal{C}_{0}$ is the line of centroid.

Let $\mathcal{C}$ denote the deformed (present) configuration at time $t$, which is determined by the position vector $\boldsymbol{R}=\boldsymbol{R}(s, t)$ and the directors $\boldsymbol{D}_{i}=\boldsymbol{D}_{i}(s, t), i=1,2,3$. The 3 unit vectors $\boldsymbol{D}_{i}$ remain mutually orthogonal after deformation, but $\boldsymbol{D}_{3}$ is no longer tangent to the curve $\mathcal{C}$, which means that the model takes into account the transverse shear deformations in rods.

We define the rotation tensor $\boldsymbol{P}$ by the relation

$$
\boldsymbol{P}(s, t)=\boldsymbol{D}_{k}(s, t) \otimes \boldsymbol{d}_{k}(s) .
$$

In (1) and throughout the paper, we employ the Einstein summation convention, and the direct tensor notation in the sense of $[44,45]$. A superposed dot denotes the time derivative as usual, while a prime designates the derivative with respect to the spatial coordinate $s$ (i.e., $f^{\prime}=\frac{\partial f}{\partial s}$ ).

We introduce the velocity vector $\boldsymbol{v}(s, t)=\dot{\boldsymbol{R}}(s, t)$ and the angular velocity vector $\boldsymbol{\omega}(s, t)$ by the wellknown relation $\dot{\boldsymbol{P}}=\boldsymbol{\omega} \times \boldsymbol{P}$, which means that $\boldsymbol{\omega}=\operatorname{axial}\left[\dot{\boldsymbol{P}} \cdot \boldsymbol{P}^{T}\right]$ is the axial vector of the antisymmetric tensor $\dot{\boldsymbol{P}} \cdot \boldsymbol{P}^{T}$.

The deformation of rods is described by two Lagrangian strain measures: the vector of extension-shear $\boldsymbol{\varepsilon}$ and the vector of bending-twisting $\phi$ is given by

$$
\boldsymbol{\varepsilon}=\boldsymbol{P}^{T} \cdot \boldsymbol{R}^{\prime}-\boldsymbol{d}_{3}, \quad \boldsymbol{\phi}=\boldsymbol{P}^{T} \cdot \operatorname{axial}\left[\boldsymbol{P}^{\prime} \cdot \boldsymbol{P}^{T}\right] .
$$

We notice that (essentially) the same strain measures have been considered in other different approaches to rod theory [4-6,38].

\section{Basic laws of thermodynamics for rods}

Let us denote by $K(s, t)$ the kinetic energy density, given by

$$
K=\frac{1}{2} \rho_{0}\left(\boldsymbol{v} \cdot \boldsymbol{v}+2 \boldsymbol{v} \cdot \boldsymbol{\Theta}_{1} \cdot \boldsymbol{\omega}+\boldsymbol{\omega} \cdot \boldsymbol{\Theta}_{2} \cdot \boldsymbol{\omega}\right),
$$


where $\rho_{0}$ is the mass density in the reference configuration $\mathcal{C}_{0}$, the second-order tensors $\boldsymbol{\Theta}_{1}(s, t)$ and $\boldsymbol{\Theta}_{2}(s, t)$ are the tensors of inertia (per unit mass), which characterize the distribution of the material in the cross-section. For the reference configuration, the tensors of inertia are denoted by $\boldsymbol{\Theta}_{1}^{0}(s)$ and $\boldsymbol{\Theta}_{2}^{0}(s)$, and we have: $\boldsymbol{\Theta}_{\alpha}=\boldsymbol{P} \cdot \boldsymbol{\Theta}_{\alpha}^{0} \cdot \boldsymbol{P}^{T}, \alpha=1,2$. We mention that the expressions of $\boldsymbol{\Theta}_{\alpha}^{0}$ in terms of the mass density of the three-dimensional rod have been determined in [9]. In view of (3), the linear momentum $\boldsymbol{K}_{1}$ and the moment of momentum $\boldsymbol{K}_{2}$ are

$$
\begin{aligned}
& \boldsymbol{K}_{1}=\frac{\partial K}{\partial \boldsymbol{v}}=\rho_{0}\left(\boldsymbol{v}+\boldsymbol{\Theta}_{1} \cdot \boldsymbol{\omega}\right) \\
& \boldsymbol{K}_{2}=\frac{\partial K}{\partial \boldsymbol{\omega}}+\boldsymbol{R} \times \frac{\partial K}{\partial \boldsymbol{v}}=\rho_{0}\left[\boldsymbol{\Theta}_{1}^{T} \cdot \boldsymbol{v}+\boldsymbol{\Theta}_{2} \cdot \boldsymbol{\omega}+\boldsymbol{R} \times\left(\boldsymbol{v}+\boldsymbol{\Theta}_{1} \cdot \boldsymbol{\omega}\right)\right] .
\end{aligned}
$$

Let $N(s, t)$ be the force vector and $\boldsymbol{M}$ be the moment vector in the rod. The balance laws of linear momentum and moment of momentum are expressed by: for any $s_{1}, s_{2} \in[0, l]$, we have

$$
\begin{aligned}
& \int_{s_{1}}^{s_{2}} \dot{\boldsymbol{K}}_{1}(s, t) \mathrm{d} s=\int_{s_{1}}^{s_{2}} \boldsymbol{F}(s, t) \mathrm{d} s+\left.\boldsymbol{N}(s, t)\right|_{s_{1}} ^{s_{2}}, \\
& \int_{s_{1}}^{s_{2}} \dot{\boldsymbol{K}}_{2}(s, t) \mathrm{d} s=\int_{s_{1}}^{s_{2}}[\boldsymbol{L}(s, t)+\boldsymbol{R}(s, t) \times \boldsymbol{F}(s, t)] \mathrm{d} s+\left.[\boldsymbol{M}(s, t)+\boldsymbol{R}(s, t) \times \boldsymbol{N}(s, t)]\right|_{s_{1}} ^{s_{2}},
\end{aligned}
$$

where $\boldsymbol{F}$ and $\boldsymbol{L}$ are the external body force and moment per unit length of $\mathcal{C}_{0}$, respectively. We mention that the assigned body loads $\boldsymbol{F}$ and $\boldsymbol{L}$ also include the contributions of the loads acting on the lateral boundaries of the three-dimensional rod [40].

To describe the thermal effects in thin rods, we consider two temperature fields: the absolute temperature field denoted by $\theta(s, t)$ and the temperature deviation $G(s, t)$, with $\theta>0$ and $G \geq 0$. In the reference configuration $\mathcal{C}_{0}$, we assume that the temperature fields $\theta_{0}, G_{0}$ are constant.

In general, the balance of energy is given by the relation

$$
\frac{\mathrm{d}}{\mathrm{d} t}(\mathcal{E}+\mathcal{K})=\mathcal{A}+\mathcal{Q}
$$

where $\mathcal{E}$ and $\mathcal{K}$ are the internal and kinetic energy functionals, respectively, $\mathcal{A}$ is the mechanical power, $\mathcal{Q}$ is the heat supply. For rods, these quantities are expressed as follows:

$$
\begin{aligned}
& \mathcal{E}=\int_{s_{1}}^{s_{2}} U \mathrm{~d} s, \quad \mathcal{K}=\int_{s_{1}}^{s_{2}} K \mathrm{~d} s \\
& \mathcal{A}=\int_{s_{1}}^{s_{2}}(\boldsymbol{F} \cdot \boldsymbol{v}+\boldsymbol{L} \cdot \boldsymbol{\omega}) \mathrm{d} s+\left.(\boldsymbol{N} \cdot \boldsymbol{v}+\boldsymbol{M} \cdot \boldsymbol{\omega})\right|_{s_{1}} ^{s_{2}}, \quad \mathcal{Q}=\int_{s_{1}}^{s_{2}} S \mathrm{~d} s+\left.q\right|_{s_{1}} ^{s_{2}}
\end{aligned}
$$

where $U(s, t)$ is the internal energy per unit length, $S$ is the external rate of the resultant heat supply per unit length, and $q$ is the heat flux along the rod. Note that $S$ consists of the internal heat supply in the rod and the heat fluxes across the rod surface.

Hence, the balance of energy is expressed by

$$
\int_{s_{1}}^{s_{2}} \frac{\mathrm{d}}{\mathrm{d} t}(K+U) \mathrm{d} s=\int_{s_{1}}^{s_{2}}(\boldsymbol{F} \cdot \boldsymbol{v}+\boldsymbol{L} \cdot \boldsymbol{\omega}+S) \mathrm{d} s+\left.(\boldsymbol{N} \cdot \boldsymbol{v}+\boldsymbol{M} \cdot \boldsymbol{\omega}+q)\right|_{s_{1}} ^{s_{2}} .
$$

We use the second law of thermodynamics (entropy inequality) in the following form:

$$
\frac{\mathrm{d}}{\mathrm{d} t} \mathcal{H} \geq \mathcal{J}
$$


where $\mathcal{H}$ is the entropy

$$
\mathcal{H}=\int_{s_{1}}^{s_{2}} \eta \mathrm{d} s
$$

$\eta(s, t)$ designates the specific entropy function, $\mathcal{J}$ is the entropy supply consisting of two parts

$$
\mathcal{J}=\int_{s_{1}}^{s_{2}} j \mathrm{~d} s+\left.h\right|_{s_{1}} ^{s_{2}},
$$

where $j$ is the resultant entropy supply, and $h$ is the entropy flux along the rod.

Following [38], we assume the following expressions for $j$ and $h$ :

$$
j=\frac{S}{\theta}-\chi G, \quad h=\frac{q}{\theta}-r G,
$$

where $\chi$ and $r$ are the additional heat supply and additional heat flux along the rod.

Hence, the second law of thermodynamics (entropy inequality) takes the following form:

$$
\int_{s_{1}}^{s_{2}} \dot{\eta} \mathrm{d} s \geq \int_{s_{1}}^{s_{2}}\left(\frac{S}{\theta}-\chi G\right) \mathrm{d} s+\left.\left(\frac{q}{\theta}-r G\right)\right|_{s_{1}} ^{s_{2}} .
$$

Using the direct approach, two temperature fields $\theta$ and $G$, as well as other 1D quantities, can be introduced only by an axiomatic way. For the interpretation of various fields in terms of three-dimensional variables, we refer to the work of Simmonds [38], who introduced previously the beamlike temperature $\theta$ and its deviation $G$. More precisely, the temperature field $\theta(s, t)$ and the temperature deviation $G(s, t)$ are identified in [38] with

$$
\frac{1}{\theta}=\frac{1}{2}\left(\frac{1}{\theta_{\min }}+\frac{1}{\theta_{\max }}\right), \quad G=\frac{1}{2}\left(\frac{1}{\theta_{\min }}-\frac{1}{\theta_{\max }}\right),
$$

where $\theta_{\min }(s, t)$ and $\theta_{\max }(s, t)$ denote the minimum and maximum values of the absolute temperature in the cross-section of the rod characterized by the spatial coordinate $s$ at time $t$. We notice that $\theta$ is the harmonic mean of $\theta_{\min }$ and $\theta_{\max }$. Using (13) is quite natural in approximation of the 3D temperature field across the rod cross-section, because in the three-dimensional entropy inequality, the temperature appears as coldness [28]. Since $G$ has the same dimension as $1 / \theta$, it can be also called the coldness deviation. If $\left(\theta_{\max }-\theta_{\min }\right) / \theta \ll 1$, then $G \simeq\left(\theta_{\max }-\theta_{\min }\right) /\left(2 \theta^{2}\right)$ and thus $G$ is related to the temperature deviation. From physical point of view, $\theta$ and $G$ describe the mean temperature at the rod cross-section and the deviation from the mean value, respectively.

\subsection{Balance equations in local forms}

Under suitable smoothness assumptions, we obtain in the classical manner [9] the following local forms of the principles (5)-(12):

- equations of motion:

$$
\begin{aligned}
& \boldsymbol{N}^{\prime}(s, t)+\boldsymbol{F}=\rho_{0} \frac{\mathrm{d}}{\mathrm{d} t}\left(\boldsymbol{v}+\boldsymbol{\Theta}_{1} \cdot \boldsymbol{\omega}\right) \\
& \boldsymbol{M}^{\prime}(s, t)+\boldsymbol{R}^{\prime} \times \boldsymbol{N}(s, t)+\boldsymbol{L}=\rho_{0}\left[\frac{\mathrm{d}}{\mathrm{d} t}\left(\boldsymbol{\Theta}_{1}^{T} \cdot \boldsymbol{v}+\boldsymbol{\Theta}_{2} \cdot \boldsymbol{\omega}\right)+\boldsymbol{v} \times \boldsymbol{\Theta}_{1} \cdot \boldsymbol{\omega}\right]
\end{aligned}
$$

- energy balance equations:

$$
q^{\prime}+P+S=\dot{U}
$$

where $P$ is given by

$$
P=N \cdot\left(\boldsymbol{v}^{\prime}+\boldsymbol{R}^{\prime} \times \boldsymbol{\omega}\right)+\boldsymbol{M} \cdot \boldsymbol{\omega}^{\prime}=(\boldsymbol{N} \cdot \boldsymbol{P}) \cdot \dot{\boldsymbol{\varepsilon}}+(\boldsymbol{M} \cdot \boldsymbol{P}) \cdot \dot{\boldsymbol{\phi}}
$$


- entropy inequality:

$$
\theta \dot{\eta} \geq S+q^{\prime}-\frac{\theta^{\prime}}{\theta} q-\chi \theta G-\theta\left(r G^{\prime}+r^{\prime} G\right),
$$

which must be valid for every point $s$ and time $t$.

Let us consider the free energy function $\Psi$ defined by

$$
\Psi=U-\theta \eta-G F,
$$

where $F$ is a new variable called the entropy deviation, according to Simmonds [34,38].

Then, the energy balance equation (15) can be written in the form

$$
q^{\prime}+S+P-\dot{\theta} \eta-\dot{G} F-\dot{\Psi}=\theta \dot{\eta}+G \dot{F} .
$$

Inserting Eq. (19) into (17), we obtain the following form of the entropy inequality:

$$
\frac{\theta^{\prime}}{\theta} q+P-\dot{\theta} \eta-\dot{G} F-\dot{\Psi} \geq\left(\dot{F}-\theta \chi-\theta r^{\prime}\right) G-\theta r G^{\prime}
$$

\section{Constitutive equations for thermoelastic rods}

For thermoelastic rods, we adopt the following constitutive assumptions: the fields $\Psi, \boldsymbol{N} \cdot \boldsymbol{P}, \boldsymbol{M} \cdot \boldsymbol{P}, \eta, F, r$ and $q$ depend only on the variables $\boldsymbol{\varepsilon}, \boldsymbol{\phi}, \theta, G, \theta^{\prime}$ and $G^{\prime}$ (and on the point $s$ ), i.e.:

$$
\{\Psi, \boldsymbol{N} \cdot \boldsymbol{P}, \boldsymbol{M} \cdot \boldsymbol{P}, \eta, F, r, q\}=\{\Psi, \boldsymbol{N} \cdot \boldsymbol{P}, \boldsymbol{M} \cdot \boldsymbol{P}, \eta, F, r, q\}\left(\boldsymbol{\varepsilon}, \boldsymbol{\phi}, \theta, G, \theta^{\prime}, G^{\prime} ; s\right) .
$$

We use the relation (21) to express $\dot{\Psi}$ into the entropy inequality (20) and we obtain

$$
\begin{gathered}
\frac{\theta^{\prime}}{\theta} q+\theta G^{\prime} r+\left(\boldsymbol{N} \cdot \boldsymbol{P}-\frac{\partial \Psi}{\partial \boldsymbol{\varepsilon}}\right) \cdot \dot{\boldsymbol{\varepsilon}}+\left(\boldsymbol{M} \cdot \boldsymbol{P}-\frac{\partial \Psi}{\partial \boldsymbol{\phi}}\right) \cdot \dot{\boldsymbol{\phi}}-\left(\eta+\frac{\partial \Psi}{\partial \theta}\right) \dot{\theta} \\
-\left(F+\frac{\partial \Psi}{\partial G}\right) \dot{G}-\frac{\partial \Psi}{\partial \theta^{\prime}} \dot{\theta}^{\prime}-\frac{\partial \Psi}{\partial G^{\prime}} \dot{G}^{\prime}+\left(\theta \chi+\theta r^{\prime}-\dot{F}\right) G \geq 0 .
\end{gathered}
$$

On the basis of Eq. (22), we deduce that

$$
\begin{gathered}
\left(\boldsymbol{N} \cdot \boldsymbol{P}-\frac{\partial(\Psi+F G)}{\partial \boldsymbol{\varepsilon}}\right) \cdot \dot{\boldsymbol{\varepsilon}}+\left(\boldsymbol{M} \cdot \boldsymbol{P}-\frac{\partial(\Psi+F G)}{\partial \boldsymbol{\phi}}\right) \cdot \dot{\boldsymbol{\phi}}-\left(\eta+\frac{\partial(\Psi+F G)}{\partial \theta}\right) \dot{\theta}-\frac{\partial(\Psi+F G)}{\partial G} \dot{G} \\
-\frac{\partial(\Psi+F G)}{\partial \theta^{\prime}} \dot{\theta}^{\prime}-\frac{\partial(\Psi+F G)}{\partial G^{\prime}} \dot{G}^{\prime}+\theta\left(\chi+r^{\prime}\right) G+\frac{\theta^{\prime}}{\theta} q+\theta G^{\prime} r \geq 0 .
\end{gathered}
$$

The standard Coleman-Noll procedure of analysis of the entropy inequality requires the independence of the rates of strains and temperature, see $[28,46]$. In our case this means that within this procedure $\dot{\boldsymbol{\varepsilon}}, \dot{\boldsymbol{\phi}}, \dot{\theta}, \dot{G}, \dot{\theta}^{\prime}, \dot{G}^{\prime}$ have to be considered as independent quantities. Assuming for a moment this independence let us apply the Coleman-Noll procedure to Eq. (23). Consider the following family of deformations:

$$
\begin{aligned}
& \boldsymbol{\varepsilon}=\boldsymbol{\varepsilon}_{0}+\boldsymbol{\varepsilon}_{1}\left(t-t_{0}\right), \quad \boldsymbol{\phi}=\boldsymbol{\phi}_{0}+\boldsymbol{\phi}_{1}\left(t-t_{0}\right), \\
& \theta=\theta_{0}+\theta_{1}\left(t-t_{0}\right)+\theta_{2}\left(s-s_{0}\right)\left(t-t_{0}\right), \\
& G=G_{0}+G_{1}\left(t-t_{0}\right)+G_{2}\left(s-s_{0}\right)\left(t-t_{0}\right),
\end{aligned}
$$

where $\boldsymbol{\varepsilon}_{0}, \boldsymbol{\varepsilon}_{1}, \boldsymbol{\phi}_{0}, \boldsymbol{\phi}_{1}, \theta_{i}, G_{i}$ are constants $(i=0,1,2), s_{0}$ is any point at the rod curve and $t_{0}$ is any time instant. Substituting Eq. (24) into Eq. (23) and taking into account that at $t=t_{0}, s=s_{0}$ we have

$$
\begin{aligned}
& \boldsymbol{\varepsilon}=\boldsymbol{\varepsilon}_{0}, \quad \dot{\boldsymbol{\varepsilon}}=\boldsymbol{\varepsilon}_{1}, \quad \boldsymbol{\phi}=\boldsymbol{\phi}_{0}, \quad \dot{\boldsymbol{\phi}}=\boldsymbol{\phi}_{1}, \\
& \theta=\theta_{0}, \quad \dot{\theta}=\theta_{1}, \quad \dot{\theta}^{\prime}=\theta_{2}, \\
& G=G_{0}, \quad \dot{G}=G_{1}, \quad \dot{G}^{\prime}=G_{2},
\end{aligned}
$$


we obtain the inequality

$$
\begin{gathered}
\left.\boldsymbol{\varepsilon}_{1} \cdot\left(\boldsymbol{N} \cdot \boldsymbol{P}-\frac{\partial(\Psi+F G)}{\partial \boldsymbol{\varepsilon}}\right)\right|_{s=s_{0}, t=t_{0}}+\left.\boldsymbol{\phi}_{1} \cdot\left(\boldsymbol{M} \cdot \boldsymbol{P}-\frac{\partial(\Psi+F G)}{\partial \boldsymbol{\phi}}\right)\right|_{s=s_{0}, t=t_{0}} \\
-\left.\theta_{1}\left(\eta+\frac{\partial(\Psi+F G)}{\partial \theta}\right)\right|_{s=s_{0}, t=t_{0}}-\left.G_{1} \frac{\partial(\Psi+F G)}{\partial G}\right|_{s=s_{0}, t=t_{0}}-\left.\theta_{2} \frac{\partial(\Psi+F G)}{\partial \theta^{\prime}}\right|_{s=s_{0}, t=t_{0}} \\
-\left.G_{2} \frac{\partial(\Psi+F G)}{\partial G^{\prime}}\right|_{s=s_{0}, t=t_{0}}+\left.\left[\left(\theta \chi+\theta \boldsymbol{r}^{\prime}\right) G+\frac{\theta^{\prime}}{\theta} q+\theta G^{\prime} r\right]\right|_{s=s_{0}, t=t_{0}} \geq 0 .
\end{gathered}
$$

The left side of the inequality (26) is a linear function of the independent variables $\boldsymbol{\varepsilon}_{1}, \boldsymbol{\phi}_{1}, \theta_{1}, \theta_{2}, G_{1}, G_{2}$. Hence, from (26) it follows

$$
\begin{aligned}
& \boldsymbol{N} \cdot \boldsymbol{P}=\frac{\partial(\Psi+F G)}{\partial \boldsymbol{\varepsilon}}, \quad \boldsymbol{M} \cdot \boldsymbol{P}=\frac{\partial(\Psi+F G)}{\partial \boldsymbol{\phi}}, \quad \eta=-\frac{\partial(\Psi+F G)}{\partial \theta}, \\
& \frac{\partial(\Psi+F G)}{\partial G}=0, \quad \frac{\partial(\Psi+F G)}{\partial \theta^{\prime}}=0, \quad \frac{\partial(\Psi+F G)}{\partial G^{\prime}}=0 \\
& \left(\theta \chi+\theta r^{\prime}\right) G+\frac{\theta^{\prime}}{\theta} q+\theta G^{\prime} r \geq 0 .
\end{aligned}
$$

From Eq. (27) it follows that the function $\widetilde{\Psi}=\Psi+F G$ does not depend on $G, \theta^{\prime}, G^{\prime}$, i.e.

$$
\widetilde{\Psi}=\widetilde{\Psi}(\varepsilon, \phi, \theta ; s),
$$

and then $\boldsymbol{M}$ does not depend on $G$. This results seems unrealistic. Indeed, from the elementary knowledge on the strength of materials we know that non-zero through-the-thickness gradient of temperature leads to the beam bending. In other words, we have $\boldsymbol{M} \neq \mathbf{0}$ if $G \neq 0$ in general, and thus $\boldsymbol{M}$ should depend on $G$. In the case of non-linear thermoelastic shells, this fact was noted in $[34,35]$ where $\dot{F}$ was considered as an internal variable with additional constitutive equation. The similar approach was used also in [38] for thermoelastic beams.

Moreover, the solution of the system of equations (27) $4,5,6$ has the form

$$
F=\frac{F_{0}(\varepsilon, \phi, \theta ; s)-\Psi}{G},
$$

where $F_{0}$ is an arbitrary function. Hence, $F$ has a singularity when $G \rightarrow 0$.

To avoid these unsatisfactory conclusions we propose a modification of Coleman-Noll procedure assuming that not all thermodynamical processes are thermodynamically admissible. More precisely, we assume that $\boldsymbol{\varepsilon}, \boldsymbol{\phi}, \theta$ can be considered as the independent variables, while $G$ is subjected to some constraints. Following our assumption let us consider the following family of deformations:

$$
\begin{aligned}
& \boldsymbol{\varepsilon}=\boldsymbol{\varepsilon}_{0}+\boldsymbol{\varepsilon}_{1}\left(t-t_{0}\right), \quad \boldsymbol{\phi}=\boldsymbol{\phi}_{0}+\boldsymbol{\phi}_{1}\left(t-t_{0}\right), \\
& \theta=\theta_{0}+\theta_{1}\left(t-t_{0}\right)+\theta_{2}\left(s-s_{0}\right)\left(t-t_{0}\right) .
\end{aligned}
$$

Substituting Eq. (28) into Eq. (22) we obtain the inequality

$$
\begin{aligned}
& \left.\boldsymbol{\varepsilon}_{1} \cdot\left(\boldsymbol{N} \cdot \boldsymbol{P}-\frac{\partial \Psi}{\partial \boldsymbol{\varepsilon}}\right)\right|_{s=s_{0}, t=t_{0}}+\left.\boldsymbol{\phi}_{1} \cdot\left(\boldsymbol{M} \cdot \boldsymbol{P}-\frac{\partial \Psi}{\partial \boldsymbol{\phi}}\right)\right|_{s=s_{0}, t=t_{0}} \\
& -\left.\theta_{1}\left(\eta+\frac{\partial \Psi}{\partial \theta}\right)\right|_{s=s_{0}, t=t_{0}}-\left.\theta_{2} \frac{\partial \Psi}{\partial \theta^{\prime}}\right|_{s=s_{0}, t=t_{0}}+\left.\left[\frac{\theta^{\prime}}{\theta} q+\theta G^{\prime} r\right]\right|_{s=s_{0}, t=t_{0}} \\
& +\left.\left[-\left(F+\frac{\partial \Psi}{\partial G}\right) \dot{G}-\frac{\partial \Psi}{\partial G^{\prime}} \dot{G}^{\prime}+\left(\theta \chi+\theta r^{\prime}-\dot{F}\right) G\right]\right|_{s=s_{0}, t=t_{0}} \geq 0
\end{aligned}
$$

From Eq. (29) it follows that

$$
\eta=-\frac{\partial \Psi}{\partial \theta}, \quad \boldsymbol{N}=\frac{\partial \Psi}{\partial \boldsymbol{\varepsilon}} \cdot \boldsymbol{P}^{T}, \quad \boldsymbol{M}=\frac{\partial \Psi}{\partial \boldsymbol{\phi}} \cdot \boldsymbol{P}^{T},
$$


while the free energy density $\Psi$ is independent of $\theta^{\prime}$. The inequality (22) reduces to the relation

$$
\frac{\theta^{\prime}}{\theta} q+\theta G^{\prime} r-\left(F+\frac{\partial \Psi}{\partial G}\right) \dot{G}-\frac{\partial \Psi}{\partial G^{\prime}} \dot{G}^{\prime}+\left(\theta \chi+\theta r^{\prime}-\dot{F}\right) G \geq 0
$$

The latter inequality can be considered as the constraint for the temperature deviation function $G$, as well as the further constraints for admissible constitutive equations for $\Psi, F, q$, and $r$. Inequality (31) implies that

$$
\frac{\partial \Psi}{\partial G^{\prime}}=0
$$

Hence, $\Psi$ takes the form $\Psi=\Psi(\boldsymbol{\varepsilon}, \boldsymbol{\phi}, \theta, G)$.

In what follows we assume the constitutive relation for $F$ in the form

$$
F=-\frac{\partial \Psi}{\partial G}
$$

which is analogue to (30) 1 . Thus, $F=F(\boldsymbol{\varepsilon}, \boldsymbol{\phi}, \theta, G)$.

The entropy inequality (31) now reduces to

$$
q \theta^{\prime}+\theta^{2} r G^{\prime}+\left(\chi+r^{\prime}-\theta^{-1} \dot{F}\right) \theta^{2} G \geq 0 .
$$

The inequality (32) should be fulfilled for any constitutive relations and any possible values of the state variables at any point of the rod. In particular, assuming $\theta^{\prime}=G^{\prime}=0$ we obtain

$$
\left[\theta\left(\chi+r^{\prime}\right)-\dot{F}\right] \theta G \geq 0 .
$$

Since $\theta>0, G \geq 0$, to satisfy this inequality we obtain that

$$
\theta\left(\chi+r^{\prime}\right)-\dot{F} \geq 0 .
$$

Introducing a new constitutive function $c=c\left(\boldsymbol{\varepsilon}, \boldsymbol{\phi}, \theta, G, \theta^{\prime}, G^{\prime}\right) \geq 0$ we transform the last relation in the form

$$
\theta\left(\chi+r^{\prime}\right)-\dot{F}=c,
$$

where $c$ should be determined by experiments, see [34-36,38]. The quantities $\chi, r$, and $F$ relate to the temperature deviation field $G$. It is quite natural to assume that $r=0, F=0$ in the case of homogeneous temperature and the absence of extra heat supply $\chi$, i.e. in the case of $G=0$ and $\chi=0$. Hence, we assume that $c=0$ when $G=0$. The simplest case is $c=c_{0} G$ with $c_{0}=$ const, which is assumed in the sequel. This case is also considered in [38].

Thus, the inequality (32) yields the results

$$
r^{\prime}+\chi-\theta^{-1} \dot{F}=c_{0} \theta^{-1} G,
$$

and

$$
q \theta^{\prime}+\theta^{2} r G^{\prime} \geq 0
$$

which is a Fourier inequality of heat conduction augmented by a term which represents heat flux in the crosssection $\Sigma$, cf. Simmonds [38]. Equation (34) plays the role of second thermoconductivity equation in the theory of thermoelastic rods and extends the analogous equation obtained in [38] where $\dot{F}$ was considered as the internal variable.

Summarizing the constitutive equations of the thermoelastic rods we have

$$
\begin{aligned}
& \Psi=\Psi(\boldsymbol{\varepsilon}, \boldsymbol{\phi}, \theta, G ; s), \\
& \eta=-\frac{\partial \Psi}{\partial \theta}, \quad F=-\frac{\partial \Psi}{\partial G}, \quad N=\frac{\partial \Psi}{\partial \boldsymbol{\varepsilon}} \cdot \boldsymbol{P}^{T}, \quad \boldsymbol{M}=\frac{\partial \Psi}{\partial \boldsymbol{\phi}} \cdot \boldsymbol{P}^{T}, \\
& \{r, q\}=\{r, q\}\left(\boldsymbol{\varepsilon}, \boldsymbol{\phi}, \theta, G, \theta^{\prime}, G^{\prime} ; s\right) .
\end{aligned}
$$


In view of the constitutive equations (36), the reduced form of the energy balance equation (19) can be written as

$$
q^{\prime}+S=\theta \dot{\eta}+G \dot{F} .
$$

The similar procedure of derivation of thermoconductivity equations for thermoelastic and thermoviscoelastic shells was proposed by Eremeyev and Pietraszkiewicz [36].

To complete the presentation of the constitutive equations we have to specify the expression for the free energy function. As an example, let us consider the following quadratic form:

$$
\begin{aligned}
\Psi= & \Psi_{0}+\boldsymbol{N}_{0} \cdot \boldsymbol{\varepsilon}+\boldsymbol{M}_{0} \cdot \boldsymbol{\phi}+\frac{1}{2} \boldsymbol{\varepsilon} \cdot \boldsymbol{A} \cdot \boldsymbol{\varepsilon}+\boldsymbol{\varepsilon} \cdot \boldsymbol{B} \cdot \boldsymbol{\phi}+\frac{1}{2} \boldsymbol{\phi} \cdot \boldsymbol{C} \cdot \boldsymbol{\phi} \\
& -\left(\boldsymbol{k}_{1} \cdot \boldsymbol{\varepsilon}\right)\left(\theta-\theta_{0}\right)-\left(\boldsymbol{k}_{2} \cdot \boldsymbol{\phi}\right)\left(\theta-\theta_{0}\right)-\left(\boldsymbol{k}_{3} \cdot \boldsymbol{\varepsilon}\right) G-\left(\boldsymbol{k}_{4} \cdot \boldsymbol{\phi}\right) G \\
& -k\left(\theta-\theta_{0}\right) G-\frac{1}{2} a\left(\theta-\theta_{0}\right)^{2}-\frac{1}{2} b G^{2},
\end{aligned}
$$

where $\Psi_{0}, k, a$ and $b$ are scalars, $\theta_{0}$ is the reference temperature, $\boldsymbol{N}_{0}, \boldsymbol{M}_{0}, \boldsymbol{k}_{1}, \boldsymbol{k}_{2}, \boldsymbol{k}_{3}, \boldsymbol{k}_{4}$ are vectors, and $\boldsymbol{A}, \boldsymbol{B}, \boldsymbol{C}$ are tensors of second order. All properties are defined in the reference configuration. The vectors $\boldsymbol{N}_{0}, \boldsymbol{M}_{0}$ describe the initial forces and moments acting in the reference configurations, $\boldsymbol{A}$ and $\boldsymbol{C}$ are the tensors of tangential and bending stiffness, respectively, $\boldsymbol{B}$ is $t$ he tensor describing the coupling between stretching and bending, $\boldsymbol{k}_{1}, \boldsymbol{k}_{2}, \boldsymbol{k}_{3}$, and $\boldsymbol{k}_{4}$ correspond to the thermoelastic coefficients.

\subsection{Structure of the constitutive tensors}

Let us choose the vectors $\boldsymbol{d}_{1}$ and $\boldsymbol{d}_{2}$ to be directed along the principal axes of inertia for any cross-section, i.e. we assume that

$$
\int_{\Sigma} \rho^{*} x_{1} \mathrm{~d} x_{1} \mathrm{~d} x_{2}=\int_{\Sigma} \rho^{*} x_{2} \mathrm{~d} x_{1} \mathrm{~d} x_{2}=0, \quad \int_{\Sigma} \rho^{*} x_{1} x_{2} \mathrm{~d} x_{1} \mathrm{~d} x_{2}=0,
$$

where $\rho^{*}$ denotes the mass density in the three-dimensional rod, and $\Sigma$ is the plane domain of the cross-section.

To determine the structure of the constitutive tensors for thermoelastic non-homogeneous rods, we employ the same method as in $[9,47,48]$, where Zhilin extends the theory of tensor symmetry to the special cases of shells and rods. For simplicity, in the remaining of this section we consider straight rods without natural twisting. In this case, we impose that the orthogonal tensor $\mathbf{1}-2 \boldsymbol{d}_{3} \otimes \boldsymbol{d}_{3}$ belongs to the symmetry group of any constitutive tensor. Then, the structure of the constitutive tensors $\boldsymbol{k}_{1}, \boldsymbol{k}_{2}, \boldsymbol{k}_{3}$ and $\boldsymbol{k}_{4}$ is given by

$$
\boldsymbol{k}_{1}=k_{1} \boldsymbol{d}_{3}, \quad \boldsymbol{k}_{2}=k_{2}^{1} \boldsymbol{d}_{1}+k_{2}^{2} \boldsymbol{d}_{2}, \quad \boldsymbol{k}_{3}=k_{3}^{1} \boldsymbol{d}_{1}+k_{3}^{2} \boldsymbol{d}_{2}, \quad \boldsymbol{k}_{4}=k_{4} \boldsymbol{d}_{3} .
$$

For the tensors $\boldsymbol{A}, \boldsymbol{B}$ and $\boldsymbol{C}$ we have the following structures

$$
\begin{aligned}
& \boldsymbol{A}=A_{1} \boldsymbol{d}_{1} \otimes \boldsymbol{d}_{1}+A_{2} \boldsymbol{d}_{2} \otimes \boldsymbol{d}_{2}+A_{3} \boldsymbol{d}_{3} \otimes \boldsymbol{d}_{3}+A_{12}\left(\boldsymbol{d}_{1} \otimes \boldsymbol{d}_{2}+\boldsymbol{d}_{2} \otimes \boldsymbol{d}_{1}\right) \\
& \boldsymbol{B}=B_{13} \boldsymbol{d}_{1} \otimes \boldsymbol{d}_{3}+B_{31} \boldsymbol{d}_{3} \otimes \boldsymbol{d}_{1}+B_{23} \boldsymbol{d}_{2} \otimes \boldsymbol{d}_{3}+B_{32} \boldsymbol{d}_{3} \otimes \boldsymbol{d}_{2}, \\
& \boldsymbol{C}=C_{1} \boldsymbol{d}_{1} \otimes \boldsymbol{d}_{1}+C_{2} \boldsymbol{d}_{2} \otimes \boldsymbol{d}_{2}+C_{3} \boldsymbol{d}_{3} \otimes \boldsymbol{d}_{3}+C_{12}\left(\boldsymbol{d}_{1} \otimes \boldsymbol{d}_{2}+\boldsymbol{d}_{2} \otimes \boldsymbol{d}_{1}\right)
\end{aligned}
$$

From the comparison of solutions (direct approach and three-dimensional approach) for non-homogeneous rods [43] we have found that the following constitutive coefficients should be taken zero:

$$
B_{13}=B_{23}=0, \quad A_{12}=0 .
$$

Inserting Eqs. (38-41) into Eq. (36) we find the explicit form of the constitutive equations for $\boldsymbol{N} \cdot \boldsymbol{P}, \boldsymbol{M} \cdot \boldsymbol{P}, \eta$ and $F$. For instance, the constitutive equations for $\eta$ and $F$ are expressed by

$$
\begin{aligned}
& \eta=k_{1} \boldsymbol{\varepsilon} \cdot \boldsymbol{d}_{3}+k_{2}^{\alpha} \boldsymbol{\phi} \cdot \boldsymbol{d}_{\alpha}+a\left(\theta-\theta_{0}\right)+k G, \\
& F=k_{3}^{\alpha} \boldsymbol{\varepsilon} \cdot \boldsymbol{d}_{\alpha}+k_{4} \boldsymbol{\phi} \cdot \boldsymbol{d}_{3}+k\left(\theta-\theta_{0}\right)+b G .
\end{aligned}
$$

From these equations we can observe the coupling of the entropy fields $\eta, F$ with the extension-shear and bending-twisting deformations $\boldsymbol{\varepsilon}, \boldsymbol{\phi}$. 
Remark The structure of the constitutive tensors can be derived also in the more general case of curved rods with natural twisting. In this case, the constitutive coefficients depend on the angle of natural twist (which is a function of $s$ ), and the expressions corresponding to (39) and (40) have to be supplemented with additional terms.

In Sects. 2-4 we have presented the basic field equations for the non-linear theory of thermoelastic rods.

\section{Linear theory of thermoelastic rods}

Let us consider the deformation of curved rods in which the displacements, rotations and variations of temperature fields are all infinitesimal. Then the rotation tensor can be represented as $\boldsymbol{P}=\mathbf{1}+\boldsymbol{\psi} \times \mathbf{1}$ within the approximation of the linear theory, where $\psi(s, t)$ is the vector of small rotations. In the linear theory, we assume that

$$
\begin{aligned}
& \boldsymbol{u}(s, t)=\boldsymbol{R}(s, t)-\boldsymbol{r}(s)=\epsilon \tilde{\boldsymbol{u}}(s, t), \quad \boldsymbol{\psi}(s, t)=\epsilon \tilde{\boldsymbol{\psi}}(s, t), \\
& T(s, t) \equiv \theta(s, t)-\theta_{0}=\epsilon \tilde{T}(s, t), \quad \tau(s, t) \equiv G(s, t)-G_{0}=\epsilon \tilde{\tau}(s, t),
\end{aligned}
$$

where $\epsilon$ is a small non-dimensional parameter such that the quantities of order $O\left(\epsilon^{2}\right)$ are neglected. The function $\tau(s, t)$ is the variation of the deviation of temperature, while $T(s, t)$ is the variation of the mean temperature. Since the absolute temperature field in the reference configuration of the three-dimensional rod is considered to be constant, then it is natural to assume that $G_{0}=0$.

The vectors of deformation become

$$
e \equiv u^{\prime}+d_{3} \times \psi=\varepsilon, \quad \kappa \equiv \psi^{\prime}=\phi,
$$

where $\boldsymbol{e}$ denotes the extension-shear vector and $\boldsymbol{\kappa}$ the bending-twisting vector in the linear theory.

The fields $\boldsymbol{N}, \boldsymbol{M}, \eta, F, q$ and $r$ are assumed to be quantities of order $O(\epsilon)$ which vanish in the reference configuration. Moreover, the free energy function $\Psi$ is a quadratic form of the arguments $\{\boldsymbol{e}, \boldsymbol{\kappa}, T, \tau\}$. The constitutive equations for $q$ and $r$ have the forms

$$
q=\varsigma T^{\prime}, \quad r=\varkappa \tau^{\prime} \text { with } \varsigma \geq 0, \varkappa \geq 0,
$$

where the scalars $\varsigma$ and $\varkappa$ characterize the thermal conductivity in the rod. Then, the entropy inequality (35) is satisfied, since it can be put in the form

$$
\varsigma\left(T^{\prime}\right)^{2}+\varkappa \theta_{0}^{2}\left(\tau^{\prime}\right)^{2} \geq 0 .
$$

The constitutive equations become

$$
\begin{aligned}
& \eta=-\frac{\partial \Psi}{\partial T}, \quad F=-\frac{\partial \Psi}{\partial \tau}, \quad N=\frac{\partial \Psi}{\partial \boldsymbol{e}}, \quad \boldsymbol{M}=\frac{\partial \Psi}{\partial \boldsymbol{\kappa}}, \\
& \Psi(\boldsymbol{e}, \boldsymbol{\kappa}, T, \tau ; s)=\frac{1}{2} \boldsymbol{e} \cdot \boldsymbol{A} \cdot \boldsymbol{e}+\boldsymbol{e} \cdot \boldsymbol{B} \cdot \boldsymbol{\kappa}+\frac{1}{2} \boldsymbol{\kappa} \cdot \boldsymbol{C} \cdot \boldsymbol{\kappa}-\left(\boldsymbol{k}_{1} \cdot \boldsymbol{e}+\boldsymbol{k}_{2} \cdot \boldsymbol{\kappa}\right) T \\
& -\left(\boldsymbol{k}_{3} \cdot \boldsymbol{e}+\boldsymbol{k}_{4} \cdot \boldsymbol{\kappa}\right) \tau-k T \tau-\frac{1}{2} a T^{2}-\frac{1}{2} b \tau^{2} .
\end{aligned}
$$

The equations of motion (14) in the linear theory are

$$
\boldsymbol{N}^{\prime}+\boldsymbol{F}=\rho_{0}\left(\ddot{\boldsymbol{u}}+\boldsymbol{\Theta}_{1}^{0} \cdot \ddot{\boldsymbol{\psi}}\right), \quad \boldsymbol{M}^{\prime}+\boldsymbol{d}_{3} \times \boldsymbol{N}+\boldsymbol{L}=\rho_{0}\left(\ddot{\boldsymbol{u}} \cdot \boldsymbol{\Theta}_{1}^{0}+\boldsymbol{\Theta}_{2}^{0} \cdot \ddot{\boldsymbol{\psi}}\right),
$$

the reduced equation of energy balance (37) becomes

$$
q^{\prime}+S=\theta_{0} \dot{\eta},
$$

while the second thermoconductivity equation (34) reduces to

$$
r^{\prime}+\chi=\theta_{0}^{-1} \dot{F}+c_{0} \theta_{0}^{-1} \tau .
$$


We present the boundary conditions and the initial conditions associated to the above field equations. Consider that the endpoints of the rod are characterized by the spatial coordinates $\bar{s}_{1}=0$ and $\bar{s}_{2}=l$. Then we take the boundary conditions of mixed type

$$
\begin{aligned}
\boldsymbol{u}\left(\bar{s}_{\gamma}, t\right)=\boldsymbol{u}^{(\gamma)}(t) & \text { or } \quad \boldsymbol{N}\left(\bar{s}_{\gamma}, t\right)=\boldsymbol{N}^{(\gamma)}(t), \\
\boldsymbol{\psi}\left(\bar{s}_{\gamma}, t\right)=\boldsymbol{\psi}^{(\gamma)}(t) & \text { or } \quad \boldsymbol{M}\left(\bar{s}_{\gamma}, t\right)=\boldsymbol{M}^{(\gamma)}(t), \\
T\left(\bar{s}_{\gamma}, t\right)=T^{(\gamma)}(t) & \text { or } \quad q\left(\bar{s}_{\gamma}, t\right)=q^{(\gamma)}(t), \\
\tau\left(\bar{s}_{\gamma}, t\right)=\tau^{(\gamma)}(t) & \text { or } \quad r\left(\bar{s}_{\gamma}, t\right)=r^{(\gamma)}(t), \quad \text { for } \gamma=1,2,
\end{aligned}
$$

and the initial conditions

$$
\begin{array}{ll}
\boldsymbol{u}(s, 0)=\boldsymbol{u}_{0}(s), & \boldsymbol{u}(s, 0)=\boldsymbol{v}_{0}(s), \quad \boldsymbol{\psi}(s, 0)=\boldsymbol{\psi}_{0}(s), \quad \dot{\boldsymbol{\psi}}(s, 0)=\boldsymbol{\omega}_{0}(s), \\
T(s, 0)=T_{0}(s), & \tau(s, 0)=\tau_{0}(s) \text { for } s \in[0, l],
\end{array}
$$

where the functions in the right-hand sides of Eqs. (49) and (50) are prescribed.

The relations (43)-(50) represent the boundary-initial-value problem for the deformation of thermoelastic rods. In view of the constitutive equations (45) and the structure of constitutive tensors (39), (40) we notice that the temperature fields are coupled also with the bending deformation of rods. This is an important advantage of considering two temperature fields for describing thermal effects in rods. In this sense, our approach is an improvement of the theory presented in [41], where the temperature field was coupled only with the extensional deformation in the linear theory of straight rods.

\subsection{Uniqueness results}

In this section we show that under certain conditions the boundary-initial-value problem of thermoelastic rods (43)-(50) admits at most one solution $\{\boldsymbol{u}, \boldsymbol{\psi}, T, \tau\}$. To this aim, we introduce the functions $\mathcal{K}(t)$ and $\mathcal{U}(t)$ by

$$
\mathcal{K}(t)=\frac{1}{2} \int_{\mathcal{C}_{0}} \rho_{0}\left(\dot{\boldsymbol{u}} \cdot \dot{\boldsymbol{u}}+2 \dot{\boldsymbol{u}} \cdot \boldsymbol{\Theta}_{1}^{0} \cdot \dot{\boldsymbol{\psi}}+\dot{\boldsymbol{\psi}} \cdot \boldsymbol{\Theta}_{2}^{0} \cdot \dot{\boldsymbol{\psi}}\right) \mathrm{d} s, \quad \mathcal{U}(t)=\int_{\mathcal{C}_{0}}(\Psi+T \eta+\tau F) \mathrm{d} s .
$$

We note that $\mathcal{K}(t)$ represents the kinetic energy of the rod, and $\mathcal{U}(t)$ can be also written in the form

$$
\mathcal{U}(t)=\int_{\mathcal{C}_{0}}\left(W+\frac{1}{2} a T^{2}+\frac{1}{2} b \tau^{2}+k T \tau\right) \mathrm{d} s,
$$

where $W$ is the strain energy function defined by

$$
W=\frac{1}{2} \boldsymbol{e} \cdot \boldsymbol{A} \cdot \boldsymbol{e}+\boldsymbol{e} \cdot \boldsymbol{B} \cdot \boldsymbol{\kappa}+\frac{1}{2} \boldsymbol{\kappa} \cdot \boldsymbol{C} \cdot \boldsymbol{\kappa} .
$$

Then, on the basis of the governing equations, we obtain by a straightforward calculation the following result.

Theorem 1 For every solution $\{\boldsymbol{u}, \boldsymbol{\psi}, T, \tau\}$ of the boundary-initial-value problem (43)-(50), the following relation holds true

$$
\begin{aligned}
\frac{\mathrm{d}}{\mathrm{d} t}[\mathcal{K}(t)+\mathcal{U}(t)]= & \int_{\mathcal{C}_{0}}\left[\left(\boldsymbol{F} \cdot \dot{\boldsymbol{u}}+\boldsymbol{L} \cdot \dot{\boldsymbol{\psi}}+\frac{1}{\theta_{0}} S T+\theta_{0} \chi \tau\right)-\frac{\boldsymbol{\zeta}}{\theta_{0}}\left(T^{\prime}\right)^{2}\right. \\
& \left.-c_{0} \tau^{2}-\theta_{0} \varkappa\left(\tau^{\prime}\right)^{2}\right] \mathrm{d} s+\left.\left(\boldsymbol{N} \cdot \dot{\boldsymbol{u}}+\boldsymbol{M} \cdot \dot{\boldsymbol{\psi}}+\frac{1}{\theta_{0}} q T+\theta_{0} r \tau\right)\right|_{0} ^{l} .
\end{aligned}
$$

Relation (54) enables us to prove the classical theorem of uniqueness stated as follows.

Theorem 2 Assume that the mass density $\rho_{0}$ is positive and the strain energy function $\mathcal{A}$ satisfies the inequality $\mathcal{A} \geq 0$. Moreover, assume that the constitutive coefficients for the thermal part: $a, b$ and $k$ verify the inequalities

$$
a>0, \quad b>0, \quad a b-k^{2}>0 .
$$

Then, the boundary-initial-value problem of thermoelastic rods has at most one solution. 
Proof If there exist two solutions of the problem then their difference, denoted also by $\{\boldsymbol{u}, \boldsymbol{\psi}, T, \tau\}$ satisfies the boundary-initial-value problem corresponding to null data. Consequently, from Theorem 1 we deduce the following relation for the difference of solutions:

$$
\frac{\mathrm{d}}{\mathrm{d} t}[\mathcal{K}(t)+\mathcal{U}(t)]+\int_{\mathcal{C}_{0}}\left[\frac{5}{\theta_{0}}\left(T^{\prime}\right)^{2}+c_{0} \tau^{2}+\theta_{0} \varkappa\left(\tau^{\prime}\right)^{2}\right] \mathrm{d} s=0
$$

From the last relation and Eq. (52) we obtain

$$
\mathcal{K}(t)+\int_{\mathcal{C}_{0}}\left(W+\frac{1}{2} a T^{2}+\frac{1}{2} b \tau^{2}+k T \tau\right) \mathrm{d} s+\int_{0}^{t} \int_{\mathcal{C}_{0}}\left[\frac{\varsigma}{\theta_{0}}\left(T^{\prime}\right)^{2}+c_{0} \tau^{2}+\theta_{0} \varkappa\left(\tau^{\prime}\right)^{2}\right] \mathrm{d} s \mathrm{~d} t=0 .
$$

Next, taking into account the inequalities $W \geq 0, \varsigma \geq 0, \varkappa \geq 0, c_{0} \geq 0$, the hypothesis (55), and the fact that $\boldsymbol{\Theta}_{2}^{0}-\boldsymbol{\Theta}_{1}^{0 T} \cdot \boldsymbol{\Theta}_{1}^{0}$ is a positive definite tensor, we deduce that

$$
\boldsymbol{u}=\mathbf{0}, \quad \boldsymbol{\psi}=\mathbf{0}, \quad T=0, \quad \tau=0 .
$$

Hence, the two solutions coincide and the proof is complete.

In what follows we show that the uniqueness result remains valid even if we drop the hypothesis $W \geq 0$. To this aim, we first prove a theorem which is the counterpart of a well-known result in classical thermoelasticity (see for example, [49], Sect. 21).

Theorem 3 Let $\mathcal{R}(t, z)$ be the function

$$
\begin{aligned}
\mathcal{R}(t, z)= & \int_{\mathcal{C}_{0}}\left(\boldsymbol{F}(t) \cdot \dot{\boldsymbol{u}}(z)+\boldsymbol{L}(t) \cdot \dot{\boldsymbol{\psi}}(z)-\frac{1}{\theta_{0}} S(t) T(z)-\theta_{0} \chi(t) \tau(z)\right) \mathrm{d} s \\
& +\left.\left(\boldsymbol{N}(t) \cdot \dot{\boldsymbol{u}}(z)+\boldsymbol{M}(t) \cdot \dot{\boldsymbol{\psi}}(z)-\frac{1}{\theta_{0}} q(t) T(z)-\theta_{0} r(t) \tau(z)\right)\right|_{0} ^{l},
\end{aligned}
$$

where $t, z \geq 0$ are two arbitrary moments of time, and the dependence on s has been omitted for brevity. Then, any solution $\{\boldsymbol{u}, \boldsymbol{\psi}, T, \tau\}$ of the boundary-initial-value problem satisfies the relation

$$
\begin{aligned}
2[\mathcal{U}(t)-\mathcal{K}(t)]= & \int_{0}^{t}[\mathcal{R}(t+\zeta, t-\zeta)-\mathcal{R}(t-\zeta, t+\zeta)] \mathrm{d} \zeta \\
& +\int_{\mathcal{C}_{0}}[\boldsymbol{N}(0) \cdot \boldsymbol{e}(2 t)+\boldsymbol{M}(0) \cdot \boldsymbol{\kappa}(2 t)+\eta(2 t) T(0)+F(2 t) \tau(0)] \mathrm{d} s \\
& -\int_{\mathcal{C}_{0}} \rho_{0}\left[\dot{\boldsymbol{u}}(2 t) \cdot\left(\dot{\boldsymbol{u}}(0)+\boldsymbol{\Theta}_{1}^{0} \cdot \dot{\boldsymbol{\psi}}(0)\right)+\dot{\boldsymbol{\psi}}(2 t) \cdot\left(\dot{\boldsymbol{u}}(0) \cdot \boldsymbol{\Theta}_{1}^{0}+\boldsymbol{\Theta}_{2}^{0} \cdot \dot{\boldsymbol{\psi}}(0)\right)\right] \mathrm{d} s .
\end{aligned}
$$

The proof of Eq. (57) can be pursued following the same procedure as in the classical case. On the basis of Theorems 1 and 3, we can prove the main uniqueness result.

Theorem 4 If the mass density $\rho_{0}$ is positive and the constitutive coefficients $a, b, k$ satisfy the conditions (55), then the boundary-initial-value problem of thermoelastic rods admits at most one solution.

Proof Assuming that there exist two solutions, it follows that their difference $\{\boldsymbol{u}, \boldsymbol{\psi}, \varphi, T\}$ verify the relations (54) and (57) corresponding to null data. Thus, from Eq. (54), we find that

$$
\mathcal{K}(t)+\mathcal{U}(t)+\int_{0}^{t} \int_{\mathcal{C}_{0}}\left[\frac{\varsigma}{\theta_{0}}\left(T^{\prime}\right)^{2}+c_{0} \tau^{2}+\theta_{0} \varkappa\left(\tau^{\prime}\right)^{2}\right] \mathrm{d} s \mathrm{~d} t=0
$$


and from Eq. (57) we get

$$
\mathcal{U}(t)-\mathcal{K}(t)=0, \quad \forall t \geq 0 .
$$

Since $\varsigma \geq 0, \varkappa \geq 0$ and $c_{0} \geq 0$, from Eqs. (58) and (59), we deduce

$$
\mathcal{U}(t)=0, \quad \mathcal{K}(t)=0, \quad \forall t \geq 0 .
$$

Finally, using the relations (52) and the hypotheses (55), we obtain that $\boldsymbol{u}=\mathbf{0}, \boldsymbol{\psi}=\mathbf{0}, T=0, \tau=0$ and the two solutions coincide.

\section{Conclusions}

In the paper, we present the thermodynamic theory of thermoelastic rods and consider the mathematical properties of the corresponding boundary-value problems. The rod is considered as a deformable Cosserat curve with two 1D temperature fields, two 1D entropy fields, and two 1D heat fluxes. These fields describe the temperature and entropy distributions on the rod cross-section. The full set of rod equations consist of two vectorial equations of motion related to the balances of linear momentum and moment of momentum, two thermoconductivity equations for the mean temperature and temperature deviation, and the reduced entropy inequality. For the linear case, we prove the uniqueness of solutions. The above results are valid for general curved rods made of thermoelastic non-homogeneous materials, which are not restricted by any material symmetry. Since these boundary-initial-value problems in the linear setting possess the desirable property of uniqueness, we may infer that the proposed thermodynamic theory for rods is well formulated.

Acknowledgments The authors acknowledge funding from the E.U. FP7 Program FP7-REGPOT-2009-1 under grant agreement no:245479, and from the Polish Ministry of Science and Higher Education, grant no:1471-1/7,PR UE/2010/7. In addition, the first author (H.A.) acknowledges support from the German Research Foundation (grant AL341/33-1) and the Japanese Society for the Promotion of Science (ID No. RC 21115001), while the second author (M.B.) was supported by the Alexander von Humboldt-Foundation. The third author (V.A.E.) was supported by the German Research Foundation (grant AL341/33-1) and by the Russian Foundation for Basic Research (grant 09-01-00459).

\section{References}

1. Todhunter, I., Pearson, K.: A History of the Theory of Elasticity and Strength of Materials, vol. I: Galilei to Saint-Venant 1639-1850, vol. II (Part I \& II): Saint Venant to Lord Kelvin, Cambridge University Press, Cambridge (1886)

2. Todhunter, I., Pearson, K.: A History of the Theory of Elasticity and Strength of Materials, vol. II (Part I \& II): Saint Venant to Lord Kelvin, Cambridge University Press, Cambridge (1893)

3. Timoshenko, S.P.: History of Strength of Materials with a Brief Account of the History of Theory of Elasticity and Theory of Structures. McGraw-Hill, New York, NY (1953)

4. Antman, S.S.: Nonlinear Problems of Elasticity. Series Applied Mathematical Sciences, vol. 107. Springer, New York, NY (1995)

5. Eliseev, V.V.: Mechanics of Elastic Bodies (in Russian). Politekhn. Univ. Publ., St. Petersburg (1996)

6. Svetlitsky, V.A.: Statics of Rods. Springer, Berlin (2000)

7. Rubin, M.B.: Cosserat Theories: Shells, Rods, and Points. Kluwer Academic Publishers, Dordrecht (2000)

8. Hodges, D.H.: Nonlinear Composite Beam Theory. Progress in Astronautics and Aeronautics, vol. 213. American Institute of Aeronautics and Astronautics Inc., Reston, VA (2006)

9. Zhilin, P.A.: Applied Mechanics-Theory of Thin Elastic Rods (in Russian). Politekhn. Univ. Publ., St. Petersburg (2007)

10. Ieşan, D.: Classical and Generalized Models of Elastic Rods. Chapman \& Hall/CRC Press, Boca Raton (2009)

11. Berdichevsky, V.L.: Variational Principles of Continuum Mechanics. II. Applications. Springer, Heidelberg (2009)

12. Cosserat, E., Cosserat, F.: Théorie des corps déformables. A. Herman et Fils, Paris (1909)

13. Ericksen, J.L., Truesdell, C.: Exact theory of stress and strain in rods and shells. Arch. Ration. Mech. Anal. 1(1), 295-323 (1958)

14. Green, A.E., Naghdi, P.M.: Non-isothermal theory of rods, plates and shells. Int. J. Solids Struct. 6, 209-244 (1970)

15. Green, A.E., Naghdi, P.M.: On thermal effects in the theory of rods. Int. J. Solids Struct. 15, 829-853 (1979)

16. DeSilva, C.N., Whitman, A.B.: Thermodynamical theory of directed curves. J. Math. Phys. 12(8), 1603-1609 (1971)

17. Kafadar, C.B.: On the nonlinear theory of rods. Int. J. Eng. Sci. 10(4), 369-391 (1972)

18. Green, A.E., Naghdi, P.M., Wenner, M.L.: On the theory of rods. II. Developments by direct approach. Proc. Roy. Soc. Lond. A. 337(1611), 485-507 (1974)

19. Beyrouthy, J., Neff, P.: A viscoelastic thin rod model for large deformations: numerical examples. Math. Mech. Solids 16(8), 887-896 (2011)

20. Irschik, H., Ziegler, F.: Thermal shock loading of elastoplastic beams. J. Therm. Stress. 8, 53-69 (1985) 
21. Irschik, H., Ziegler, F.: Dynamic analysis of elastic-plastic beams by means of thermoelastic solutions. Int. J. Solids Struct. 21, 819-829 (1985)

22. Fotiu, P., Irschik, H., Ziegler, F.: Forced vibrations of an elasto-plastic and deteriorating beam. Acta Mechanica 69, 193-203 (1987)

23. Irschik, H.: Analogy between refined beam theories and the Bernoulli-Euler theory. Int. J. Solids Struct. 28, $1105-1112(1991)$

24. Irschik, H.: On vibrations of layered beams and plates. ZAMM 73, T34-T45 (1993)

25. Krommer, M., Irschik, H.: On the influence of the electric field on free transverse vibrations of smart beams. Smart Mater. Struct. 8, 401-410 (1999)

26. Irschik, H.: A review on static and dynamic shape control of structures by piezoelectric actuation. Eng. Struct. 24, 5-11 (2002)

27. Hummer, A., Irschik, H.: Large deformation and stability of an extensible elastica with an unknown length. Int. J. Solids Struct. 48(11), 1301-1310 (2011)

28. Truesdell, C.: Rational Thermodynamics. 2nd edn. Springer, New York (1984)

29. Maugin, G.A.: Internal variables and dissipative structures. J. Non-Equilib. Thermodyn. 15, 173-192 (1990)

30. Maugin, G.A., Muschik, W.: Thermodynamics with internal variables, part I: general concepts, part II: applications. J. Non-Equilib. Thermodyn. 19, 217-249, 250-289 (1994)

31. Muschik, W., Papenfuss, C., Ehrentraut, H.: A sketch of continuum thermodynamics. J. Non-Newton. Fluid Mech. 96(1-2, Sp. Iss. SI), 255-290 (2001)

32. Muschik, W.: Survey of some branches of thermodynamics. J. Non-Equilib. Thermodyn. 33(2), 165-198 (2008)

33. Berezovski, A., Engelbrecht, J., Maugin, G.A.: Generalized thermomechanics with dual internal variables. Arch. Appl. Mech. 81, 229-240 (2011)

34. Simmonds, J.G.: The thermodynamical theory of shells: descent from 3-dimensions without thickness expansions. In: Axelrad, E.K., Emmerling, F.A. (eds.) Flexible Shells, Theory and Applications, pp. 1-11. Springer, Berlin (1984)

35. Simmonds, J.G.: A classical, nonlinear thermodynamic theory of elastic shells based on a single constitutive assumption. J. Elast. 105(1-2), 305-312 (2011)

36. Eremeyev, V.A., Pietraszkiewicz, W.: Thermomechanics of shells undergoing phase transition. J. Mech. Phys. Solids 59, 1395-1412 (2011)

37. Pietraszkiewicz, W.: Refined resultant thermomechanics of shells. Int. J. Eng. Sci. 49(10), 1112-1124 (2011)

38. Simmonds, J.G.: A simple nonlinear thermodynamic theory of arbitrary elastic beams. J. Elast. 81, 51-62 (2005)

39. Zhilin, P.A.: Nonlinear theory of thin rods. In: Indeitsev, D.A., Ivanova, E.A., Krivtsov, A.M. (eds.) Advanced Problems in Mechanics, vol. 2., pp. 227-249. Instit. Problems Mech. Eng. R.A.S. Publ., St. Petersburg (2006)

40. Bîrsan, M., Altenbach, H.: On the theory of porous elastic rods. Int. J. Solids Struct. 48, 910-924 (2011)

41. Bîrsan, M., Altenbach, H.: Theory of thin thermoelastic rods made of porous materials. Arch. Appl. Mech. 81(10), 1365-1391 (2011)

42. Ieşan, D.: A theory of thermoelastic materials with voids. Acta Mech. 60, 67-89 (1986)

43. Bîrsan, M., Altenbach, H., Sadowski, T., Eremeyev, V.A., Pietras, D.: Deformation analysis of functionally graded rods by the direct approach. Compos. Part B (2011). doi:10.1016/j.compositesb.2011.09.003

44. Lurie, A.I.: Theory of Elasticity. Springer, Berlin (2005)

45. Lebedev, L.P., Cloud, M.J., Eremeyev, V.A.: Tensor Analysis with Applications in Mechanics. World Scientific, New Jersey (2010)

46. Coleman, B.D., Noll, W.: The thermodynamics of elastic materials with heat conduction and viscosity. Arch. Ration. Mech. Anal. 13, 167-178 (1963)

47. Zhilin, P.A.: Applied Mechanics-Foundations of Shell Theory (in Russian). Politekhn. Univ. Publ., St. Petersburg (2006)

48. Altenbach, H., Naumenko, K., Zhilin, P.A.: A direct approach to the formulation of constitutive equations for rods and shells. In: Pietraszkiewicz, W., Szymczak, C. (eds.) Shell Structures: Theory and Applications, pp. 87-90. Taylor and Francis, London (2006)

49. Carlson, D.E.: Linear thermoelasticity. In: Flügge, W. (ed.) Handbuch der Physik, vol. VIa/2, pp. 297-346. Springer, Berlin (1972) 